Check for updates

Cite this: RSC Adv., 2018, 8, 22161

\title{
Biochar supported sulfide-modified nanoscale zero-valent iron for the reduction of nitrobenzene $\uparrow$
}

\author{
Dejin Zhang, Yang Li, Siqi Tong, Xinbai Jiang, * Lianjun Wang, Xiuyun Sun, Jiansheng Li, \\ Xiaodong Liu and Jinyou Shen (iD) *
}

Sulfide-modified nanoscale zerovalent iron (S-nZVI) was effectively utilized for the reduction of various contaminants, despite its applicability being limited due to agglomeration, oxidation and electron loss. In this study, biochar (BC)-supported S-nZVI was prepared to enhance the reactivity of S-nZVI for nitrobenzene (NB) reduction. Scanning electron microscopy images showed that the S-nZVI particles were well-dispersed on the $B C$ surface as well as in the channels. NB removal and aniline formation could be significantly enhanced by using S-nZVI@BC, as compared to S-nZVI and blank BC. NB removal by S-nZVI@BC followed the pseudo second-order kinetics model and Langmuir isotherm model, suggesting hybrid chemical reaction-sorption was involved. Furthermore, a possible reaction mechanism for enhanced NB removal by S-nZVI@BC was proposed, including chemical adsorption of NB onto S-nZVIaBC, direct reduction by S-nZVI and enhanced electron transfer. The high reducibility of S-nZVI@BC as well as its excellent antioxidation ability and reusability demonstrated its promising prospects in remediation applications.

Received 21st May 2018

Accepted 6th June 2018

DOI: $10.1039 / \mathrm{c} 8 \mathrm{ra04314k}$

rsc.li/rsc-advances

solubility of $\mathrm{AgS}_{2}$. Furthermore, S-nZVI can be produced following a single-step synthetic procedure with relative ease as compared to other nZVI-based materials. Kim et al. ${ }^{7}$ have reported that the $\mathrm{Fe} / \mathrm{FeS}$ nanoparticles could be produced through the addition of dithionite during the synthesis of nZVI via borohydride reduction of ferric chloride. The obtained Fe/ FeS nanoparticles showed higher reactivity toward TCE than nZVI. Enhanced cadmium removal by S-nZVI through reduction, adsorption and co-precipitation has been reported by $\mathrm{Su}$ et al. ${ }^{\mathbf{8}}$ where S-nZVI was synthesized in a similar manner.

However, the fine sized nZVI-based materials are unstable and can aggregate easily, which may limit its dispersibility in aqueous solutions and present challenges for the continuous in situ environmental remediation. ${ }^{9}$ The oxidation of S-nZVI can diminish its reactivity by the reason that the formation of oxide layers blocked the serviceable reactive sites. Additionally, loss of extra electrons from S-nZVI reduces its electron transfer efficiency, resulting in lower target contaminant removal capacity. Therefore, attempts should be made to improve reduction capacity and electron transfer efficiency of S-nZVI. Loading of nZVI onto support materials such as activated carbon, ${ }^{10}$ clay $^{11}$ and resin ${ }^{12}$ could prevent the aggregation of nZVI and enhance its reduction efficiency. Theoretically and practically, the S-nZVI particles could also be loaded onto these support materials for better dispersion and higher reactivity. Nonetheless, efficient support material for S-nZVI particles has never been revealed previously.

Biochar (BC) is a porous carbon material formed by the thermal conversion of cost-optimal raw biomass, particularly the lignocellulosic waste. ${ }^{13}$ It is commonly used as an adsorbent material in environmental remediation owing to its highly

Jiangsu Key Laboratory of Chemical Pollution Control and Resources Reuse, School of Environmental and Biological Engineering, Nanjing University of Science and Technology, Nanjing 210094, Jiangsu Province, China. E-mail: xinbai_jiang@njust. edu.cn; shenjinyou@mail.njust.edu.cn; Fax: +86 25 84315941; +86 25 84303965; Tel: +862584315941; +862584303965

$\dagger$ Electronic supplementary information (ESI) available. See DOI: 10.1039/c8ra04314k 
developed porosity, excellent adsorption capacity and high surface reactivity. ${ }^{\mathbf{1 4}}$ Various contaminants can be effectively adsorbed onto BC through interaction via van der Waals forces, electrostatic attraction or chemical bonds. ${ }^{15}$ Due to its multifunctional features, BC has been employed as a support material for the dispersion and stabilization of engineered nanoparticles to improve their environmental application. Ahmed et $a{ }^{16}{ }^{16}$ have previously reported enhanced chloramphenicol removal by nZVI immobilized onto functionalized biochar (nZVI-fBC). They found that nZVI-fBC could undergo a quick reaction to form magnetic-biochar composite $\left(n \mathrm{Fe}_{3} \mathrm{O}_{4}-\mathrm{fBC}\right)$, rendering its ability of both reduction and sorption. It is therefore useful to explore the synergistic effect between S-nZVI and $\mathrm{BC}$ by combining the advantages of both. However, very limited research has been done to address such an intriguing topic.

Herein, the composite S-nZVI@BC was synthesized and characterized in this study. Its performance in the removal of nitrobenzene (NB) was investigated and compared with the bare S-nZVI and blank BC. The underlying mechanisms were elucidated based on NB removal kinetics and isotherm, identification of corrosion products and electrochemical analysis.

\section{Materials and methods}

\subsection{Preparation of S-nZVI@BC composite materials}

$\mathrm{BC}$ was prepared using rice husk as raw material, following the method developed in our previous study. ${ }^{17}$ S-nZVI was synthesized using dithionite at room temperature, following the method described by Su et al. ${ }^{8}$ BC supported S-nZVI composites were prepared according to the following procedure. Specifically, $0.63 \mathrm{~g} \mathrm{BC}$ and $8 \mathrm{~g} \mathrm{FeSO}_{4} \cdot 7 \mathrm{H}_{2} \mathrm{O}$ were mixed in $100 \mathrm{~mL}$ deionized water in a three-necked flask. Then the mixture in the flask were stirred for $20 \mathrm{~min}$. Subsequently, $100 \mathrm{~mL}$ freshly prepared solution containing $7.6 \mathrm{~g} \mathrm{NaBH}_{4}$ and $0.75 \mathrm{~g} \mathrm{Na} \mathrm{S}_{2} \mathrm{O}_{4}$ was added dropwise into the mixture with vigorous stirring under $\mathrm{N}_{2}$ atmosphere for $1 \mathrm{~h}$. After the reduction was complete, the resulting precipitate was collected using a magnet, washed five times with deionized water and twice with deoxygenated ethanol. Thereafter, the precipitate was dried in oven at $50{ }^{\circ} \mathrm{C}$ under vacuum overnight and stored in sealed vials in an anaerobic glovebox for further use. The resulting molar ratio of $\mathrm{S} / \mathrm{Fe}$ was 0.3 and the mass ratio of S-nZVI/BC was 3.

\subsection{NB removal by S-nZVI@BC}

NB removal performance of the synthesized S-nZVI/BC was assessed through batch experiment, which were conducted in $125 \mathrm{~mL}$ serum bottles containing $100 \mathrm{~mL} 100 \mathrm{mg} \mathrm{L}^{-1} \mathrm{NB}$ solution with the addition of $0.1 \mathrm{~g}$ S-nZVI@BC at pH 7.0. For comparison, NB removal by S-nZVI alone and BC alone was also investigated in the same manner. The mixture was shaken on a horizontal vibrator at $200 \mathrm{rpm}$ and $35{ }^{\circ} \mathrm{C}$ in dark. The headspace of the bottles was purged with $\mathrm{N}_{2}$ to eliminate the interference of oxygen. In order to evaluate the NB removal efficiency, NB removal profile was described by pseudo firstorder and second-order models. ${ }^{18}$ Langmuir, Freundlich and
Redlich-Peterson models were used for simulating the removal isotherms. ${ }^{19}$ Both kinetics and isotherms analysis were investigated within the initial NB concentration range of 100$700 \mathrm{mg} \mathrm{L}^{-1}$ at $\mathrm{pH}$ 7.0. In order to avoid significant adsorption of NB and its degradation intermediates by S-nZVI@BC, especially at low initial NB concentration, S-nZVI@BC dosage adopted in kinetics and isotherms analysis was controlled at $0.2 \mathrm{~g} \mathrm{~L}^{-1}$.

In order to further compare the performance of S-nZVI and SnZVI@BC for NB removal, aging effect was examined with $S$ nZVI and S-nZVI@BC particles exposed to air for 1, 5, 8, 10 and 12 weeks. To investigate their reusability, S-nZVI and SnZVI@BC particles were reacted with NB for four repetitive cycles. The particles were recovered through centrifugation at $8000 \mathrm{rpm}$ for $10 \mathrm{~min}$. The recovered particles were added into fresh NB solution $\left(100 \mathrm{mg} \mathrm{L}^{-1}\right)$ under the same experimental condition to evaluate their performance of NB removal.

\subsection{Analytical methods}

SEM equipped with an energy dispersive X-ray spectroscopy (EDS) was used to determine the morphological and chemical composition of the materials (Quant ${ }^{\mathrm{TM}} 250$ FEG, Thermo Fisher Scientific Inc., USA). The Brunauer-Emmett-Teller (BET) specific surface area was determined by the $\mathrm{N}_{2}$ adsorption method using a TriStar 3000 system (Micromeritics Corp., USA). XRD analysis was carried out on a Bruker-AXSD8 system (Bruker AXS Inc., Germany) with $\mathrm{Cu}-\mathrm{K} \alpha$ radiation source operating at $40 \mathrm{kV}$ and 40 mA. FTIR spectroscopy (Nicolet iS10, Thermo Fisher Scientific Inc., USA) was used for identifying the functional groups and the chemical bonding of the composites within wavenumber range of $4000-400 \mathrm{~cm}^{-1}$. The chemical states of the surface elements were recorded by XPS with $\mathrm{Al} \mathrm{K} \alpha$ radiation at $1486.8 \mathrm{kV}$ on a $\mathrm{PHI}$ Quanera II ESCA System (Physical Electronics, Inc., USA). Electrochemical analysis of the composites was performed with an electrochemical workstation (Bio-Logic Science Instruments, VMP3, France), following Li et al. ${ }^{1}$

At predetermined time intervals, the aqueous samples were withdrawn from the bottle and passed through a $0.22 \mu \mathrm{m}$ filter for further analysis. Both NB and aniline (AN) were quantified by high performance liquid chromatography (HPLC, LC-20, Shimazu, Japan) equipped with a RP18 column $(5 \mu \mathrm{m}, 4.6 \mathrm{~mm} \times 250 \mathrm{~mm})$. The mobile phase of water-methanol $(60 / 40, \mathrm{v} / \mathrm{v})$ was set to flow at

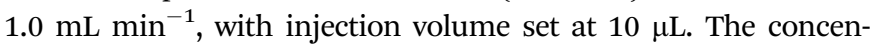
tration of ferrous ions was determined by 1,10-phenanthroline method using an UV-vis spectrometer (LAMBDA 750, PerkinElmer, USA) at $510 \mathrm{~nm}$. All analysis was conducted in triplicate and the data was reported as the arithmetic mean \pm standard deviation.

\section{Results and discussion}

\subsection{Characterization of S-nZVI@BC particles}

The morphologies of the BC, S-nZVI and S-nZVI@BC samples were characterized by SEM. Blank BC looked like a small hive with a smooth surface and porous shape (Fig. 1a). The synthesized S-nZVI particles were roughly globular and aggregated into a chain-like conformation with particle sizes lying in the nanoscale range (Fig. 1b). S-nZVI@BC showed clearly defined 

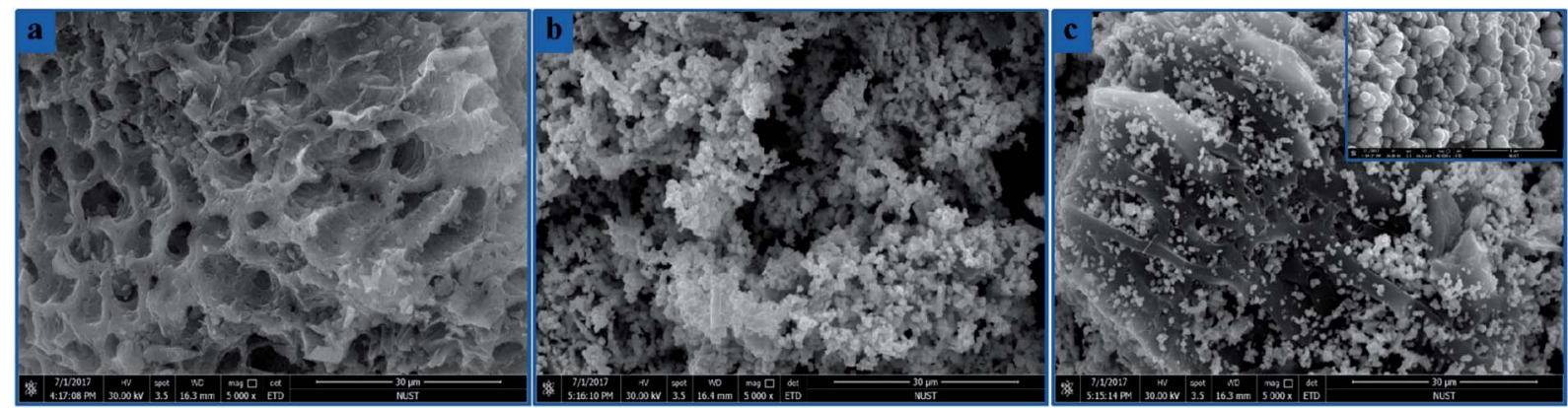

Fig. 1 SEM images of blank BC (a), S-nZVI (b) and S-nZVI@BC (c). The inset of S-nZVI@BC with resolution of $4 \mu \mathrm{m}$.

and discrete S-nZVI particles on the BC surface as well as in its channels (Fig. 1c). The synthesized S-nZVI particles on SnZVI@BC showed a globular and furry structure with the diameter ranging from 200 to $950 \mathrm{~nm}$. According to the previous studies, pristine S-nZVI showed flake-like shell structure and the core did not have an obvious compact shell. ${ }^{8}$ The surface area of S-nZVI@BC $\left(61.03 \mathrm{~m}^{2} \mathrm{~g}^{-1}\right)$ was significantly lower than that of BC $\left(120.25 \mathrm{~m}^{2} \mathrm{~g}^{-1}\right)$, which could be attributed to the occupation or even partial blocking of S-nZVI in BC channels.

In order to further understand the elemental distribution on the S-nZVI@BC particles, mapping analysis was conducted (Fig. S1 $\dagger$ ). The EDS spectra of selected zone showed O/C atomic ratio of 0.23 , representing a typical $\mathrm{BC}$ matrix. ${ }^{20}$ High Fe and S contents of $64.7 \pm 1.2 \%$ and $1.1 \pm 0.1 \%$ suggested the successful impregnation of $\mathrm{Fe}$ and $\mathrm{S}$ onto $\mathrm{BC}$ surface. The phase composition of BC, S-nZVI and S-nZVI@BC was evaluated by XRD characterization, as shown in Fig. 2. BC displayed a diffraction peak at $22.0^{\circ}$, corresponding to a layer-to-layer distance ( $d$-spacing) of $0.40 \mathrm{~nm}$. The large $d$-spacing of the BC was attributed to the presence of the $-\mathrm{OH}, \mathrm{O}=\mathrm{C}-\mathrm{O}$ and $\mathrm{C}-\mathrm{O}$ groups. ${ }^{18}$ The broad XRD reflection peaks suggested that nZVI was present within the synthesized S-nZVI and S-nZVI@BC, as shown by the two sharp $2 \theta$ peaks at 44.68 and $65.03^{\circ}$, which corresponded to the [110] and [200] directions of $\alpha$-Fe. ${ }^{21}$

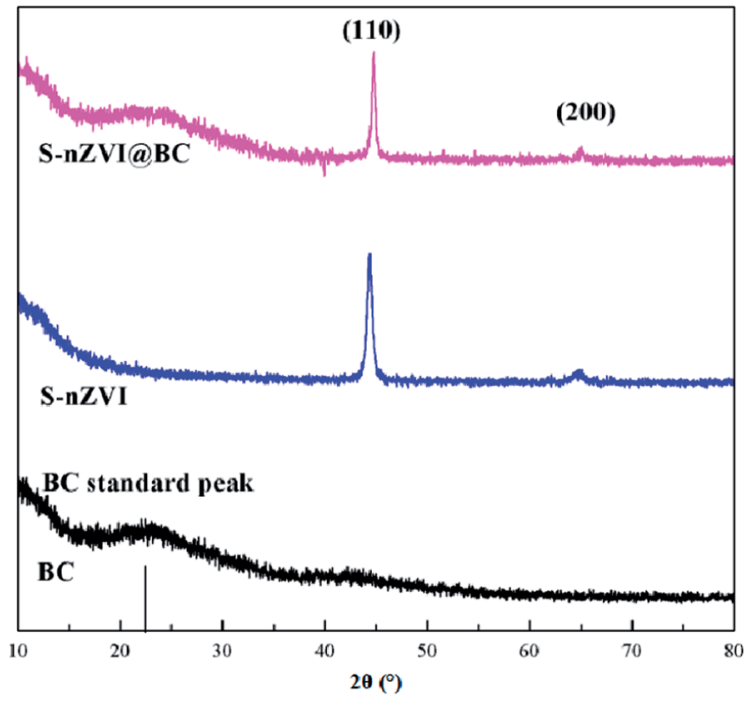

Fig. 2 XRD patterns of blank BC, S-nZVI and S-nZVI@BC.
However, no clear peaks related to sulfur-bearing compounds such as FeS or $\mathrm{FeS}_{2}$ could be observed in the diffraction patterns of both S-nZVI and S-nZVI@BC, probably due to the low concentration or low crystallinity degree of sulfur-bearing compounds. Similar phenomenon has been reported with regard to the XRD data of the precipitated iron sulfides by $\mathrm{Su}$ et $a .^{8}$ The surface functional groups on the S-nZVI@BC were further analyzed by FTIR (Fig. S2 $\dagger$ ). The spectrum of the SnZVI@BC composite exhibited conspicuous peak at $576 \mathrm{~cm}^{-1}$, which might be attributed to the Fe-S bond, ${ }^{22}$ suggesting that iron sulfides (i.e., FeS or $\mathrm{FeS}_{2}$ ) could be formed on the nZVI surface. It was intuitive that the electron transfer efficiency between S-nZVI and the contaminants could be enhanced due to the excellent electrical conductivity of iron sulfides. ${ }^{1}$

\subsection{Application of S-nZVI@BC for NB removal}

In order to investigate the reduction performance of the asprepared S-nZVI@BC, NB was chosen as the model contaminant. Under reductive condition, NB could be transformed into the final reduction product, i.e., AN, with nitrosobenzene and hydroxylaminobenzene as the main intermediates. ${ }^{23}$ From HPLC analysis of the supernatant for S-nZVI (Fig. S3a†), four peaks could be observed at retention time of 5.00, 5.85, 14.93 and $18.56 \mathrm{~min}$, which were identified as hydroxylaminobenzene, AN, NB and nitrosobenzene based on authentic standard and UV-vis analysis. However, only AN could be observed in the HPLC chromatogram of the supernatant for S-nZVI@BC during the whole degradation period (Fig. S3b†), confirming more thorough reduction of NB by S-nZVI@BC.

As shown in Fig. 3a, $60.2 \pm 3.1 \%$ of $100 \mathrm{mg} \mathrm{L}^{-1} \mathrm{NB}$ could be removed by S-nZVI@BC within degradation time as short as $10 \mathrm{~min}$, as compared to the low NB removal efficiency of only 5.1 $\pm 0.2 \%$ by S-nZVI within the same period. In the S-nZVI@BC degradation system, complete NB removal was observed after $30 \mathrm{~min}$, whereas with the addition of S-nZVI and BC, $22.4 \pm$ $0.9 \%$ and $68.6 \pm 1.2 \%$ of NB remained in the supernatant even after $120 \mathrm{~min}$. Correspondingly, in the degradation system by SnZVI@BC, AN formation efficiency increased from 13.0 $\pm 0.8 \%$ at $10 \mathrm{~min}$ to $84.6 \pm 1.7 \%$ at $120 \mathrm{~min}$, which was significantly higher than those in the systems by S-nZVI or BC at any given degradation time (Fig. 3b). The rather high NB removal and AN formation efficiency observed in this study indicated excellent reduction performance of S-nZVI@BC. 

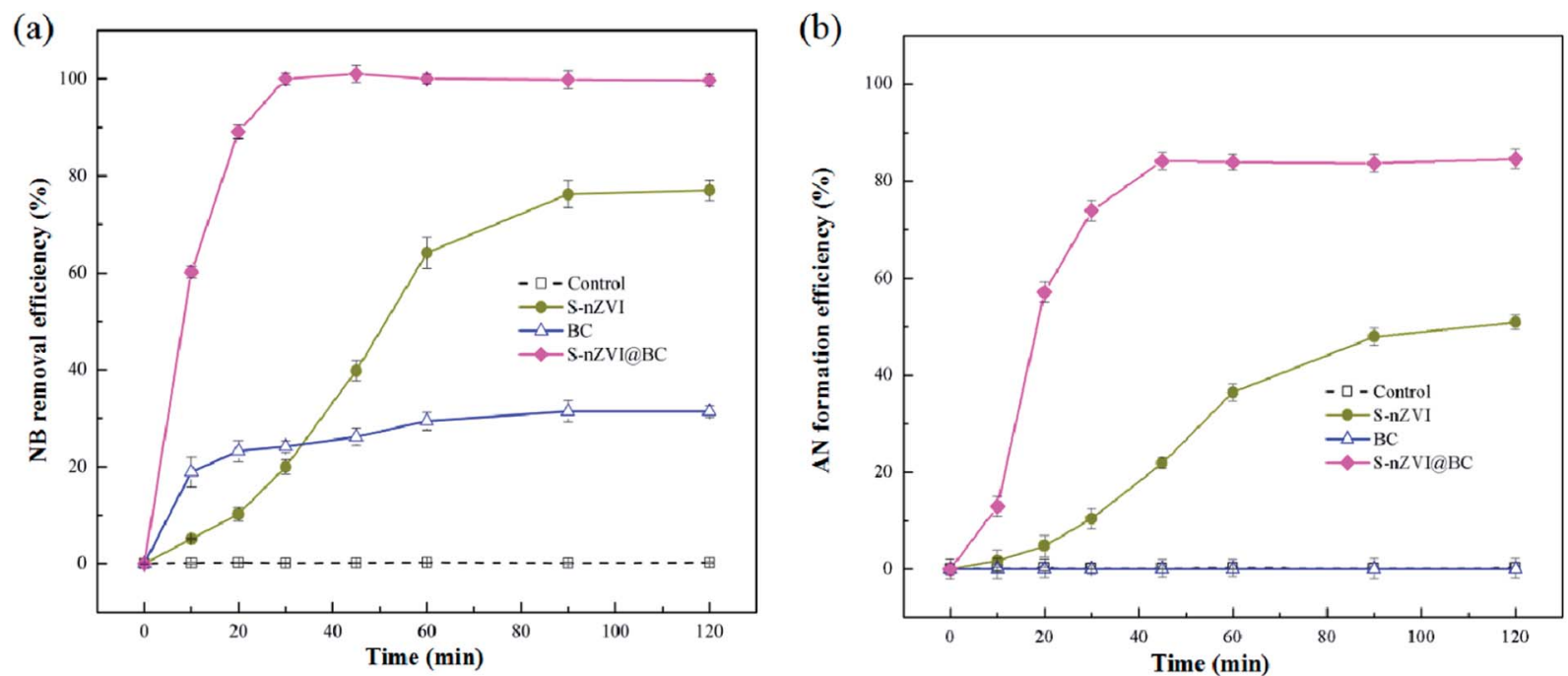

Fig. 3 The overall performance of blank BC, S-nZVI and S-nZVIaBC in terms of NB removal (a) and AN formation (b).

\subsection{NB removal kinetics and isotherm}

Fig. 4a showed the profile of NB removal by S-nZVI@BC particles at different initial NB concentrations. As indicated in Fig. 4a, NB removed by S-nZVI@BC increased rapidly within the first $30 \mathrm{~min}$, followed by a slow step until $360 \mathrm{~min}$. According to the literature, NB removal by nZVI-based materials often underwent a multi-step process that involves electrostatic adsorption and subsequent reduction of $\mathrm{NB}^{21}$ In order to describe the NB removal profile by S-nZVI@BC, pseudo firstorder and second-order kinetic models were used to fit the kinetic data. As indicated in Table S1, $\uparrow$ the pseudo second-order kinetic model fit the data better $\left(R^{2}>0.95\right)$ compared to pseudo first-order reaction model. This phenomenon indicated that the rate-limiting process for NB removal by S-nZVI@BC particles was chemical process, which involved either sharing or exchanging of electrons between NB and S-nZVI@BC. ${ }^{24}$

Three common isotherm models, namely Langmuir, Freundlich and Redlich-Peterson, were used to describe the NB removal behavior of S-nZVI@BC. ${ }^{19}$ As shown in Fig. 4b, the NB removal profile could be well fitted by the Langmuir model, whose correlation coefficient $R^{2}$ value was as high as 0.9985 . In contrast, the Freundlich and Redlich-Peterson model were not suitable to describe the NB removal profile with a relative lower correlation coefficient $R^{2}$ (Table $\mathrm{S} 2 \dagger$ ). The good fitting of Langmuir isotherm model indicated that NB removal by S-nZVI@BC mainly occurred homogeneously on the surface via monolayer sorption. ${ }^{25}$ The calculated $q_{\mathrm{m}}$ value representing the NB removal capacity of S-nZVI@BC, was as high as $588.23 \mathrm{mg} \mathrm{g}^{-1}$, which was significantly higher than magnetic biochar $\left(38.38 \mathrm{mg} \mathrm{g}^{-1}\right) .^{26}$

\subsection{Identification of S-nZVI@BC corrosion products}

S-nZVI@BC after reaction with $100 \mathrm{mg} \mathrm{L}^{-1} \mathrm{NB}$ for four times was further characterized by SEM, FTIR, XRD and XPS. As indicated by SEM images in Fig. S1, $\uparrow$ S-nZVI@BC aggregated while the surface became coarse after reaction with NB. EDS results indicated that the atomic ratio of elemental $\mathrm{Fe}$ and $\mathrm{S}$ decreased, whereas that of O increased simultaneously, which could be attributed to the consumption of Fe and $\mathrm{S}$ as well as
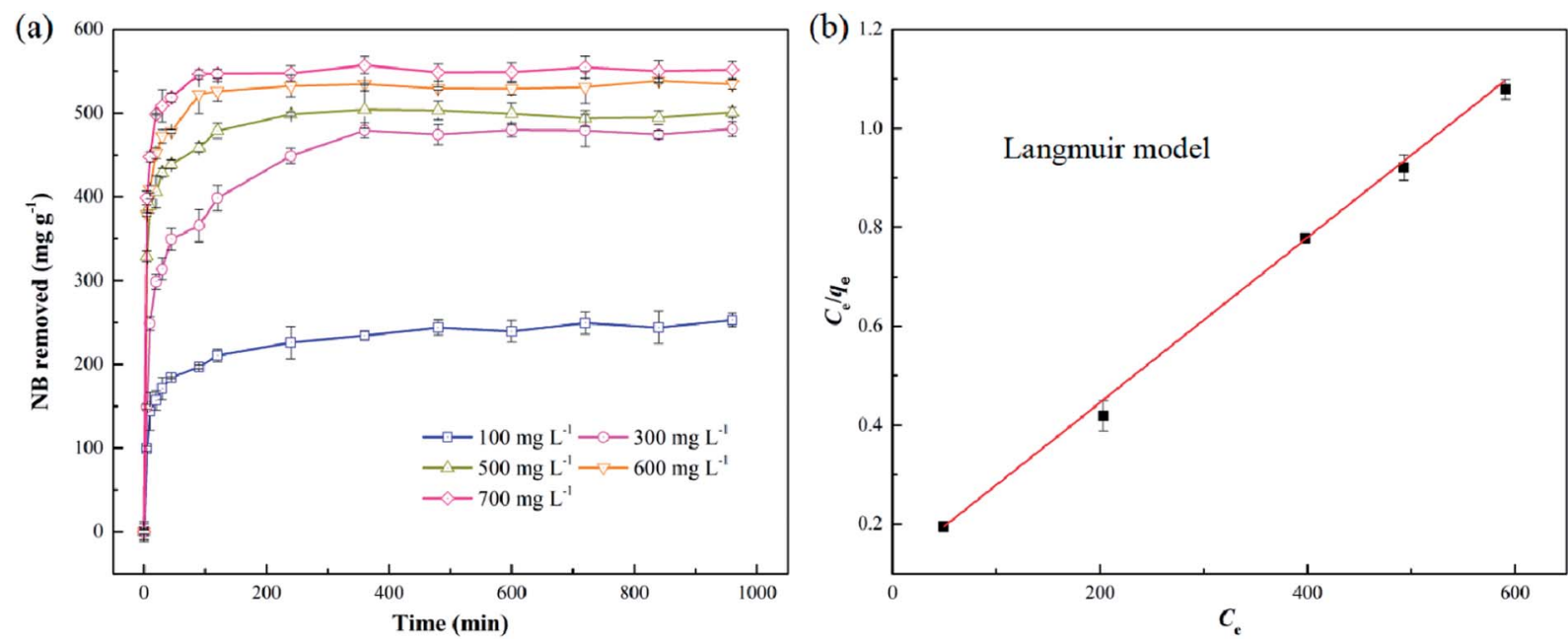

Fig. 4 NB removal kinetics model (a) and Langmuir isotherm model (b). 
the formation and deposition of iron oxides on the surface of SnZVI@BC. FTIR results indicated that $\mathrm{Fe}-\mathrm{S}$ vibration at $576 \mathrm{~cm}^{-1}$ disappeared, while new peaks at $698 \mathrm{~cm}^{-1}, 1133 \mathrm{~cm}^{-1}$ and $1196 \mathrm{~cm}^{-1}$ were observed after reaction (Fig. S2†), which could be assigned to the $\mathrm{Fe}-\mathrm{O}$ vibration and $\mathrm{S}-\mathrm{O}$ vibration. ${ }^{27}$ The observed $-\mathrm{OH}$ stretching band shifted from $3307 \mathrm{~cm}^{-1}$ for fresh particles to $3674 \mathrm{~cm}^{-1}$ for particles after reaction, which could be attributed to the enhanced interaction between $-\mathrm{OH}$ and $\mathrm{Fe}^{28}$ The XRD spectrum of S-nZVI@BC after reaction (Fig. S4 $\dagger$ ) confirmed that iron oxides could be formed as a result of iron corrosion..$^{29,30}$

In order to further clarify the formation of iron corrosion products, XPS survey was performed for Fe and S species on SnZVI@BC. As shown in Fig. 5, Fe spectrum showed four peaks, including the peak located at $706.7 \mathrm{eV}$ related to nZVI species, ${ }^{31}$ the peak at $710.6 \mathrm{eV}\left(\mathrm{Fe} 2 \mathrm{p}_{3 / 2}\right)$ attributed to $\mathrm{Fe}(\mathrm{II})$ and peaks at $712.6 \mathrm{eV}\left(\mathrm{Fe} 2 \mathrm{p}_{3 / 2}\right)$ and $724.5 \mathrm{eV}\left(\mathrm{Fe} 2 \mathrm{p}_{1 / 2}\right)$ assigned to $\mathrm{Fe}(\mathrm{III}) .{ }^{1,32}$ The peak at $710.6 \mathrm{eV}$ corresponded to the Fe(II) state in FeS, while the peaks at $712.6 \mathrm{eV}$ and $724.5 \mathrm{eV}$ were on behalf of iron oxides. ${ }^{33}$ Notably, after reaction with NB, peaks corresponding to nZVI and Fe(II) were weakened, while peaks corresponding to $\mathrm{Fe}(\mathrm{III})$ was strengthened. The $\mathrm{S} 2 \mathrm{p}$ spectrum of SnZVI@BC showed three peaks at $160.8 \mathrm{eV}, 162.0 \mathrm{eV}$ and $168.0 \mathrm{eV}$, which could be assigned to the $\mathrm{S}^{2-}$, FeS and $\mathrm{SO}_{4}{ }^{2-}$ species, respectively ${ }^{21}$ After reaction with $\mathrm{NB}$, the $\mathrm{S} 2 \mathrm{p}$ spectrum showed four peaks at $161.4 \mathrm{eV}, 162.1 \mathrm{eV}, 163.0 \mathrm{eV}$ and $168.7 \mathrm{eV}$, which corresponded to the $\mathrm{S}^{2-}, \mathrm{FeS}, \mathrm{S}_{n}{ }^{2-}$ and $\mathrm{SO}_{4}{ }^{2-}$ groups, respectively, validating the oxidation of sulfides at the presence of NB. ${ }^{7}$

\subsection{Antioxidation activity and reusability of S-nZVI@BC}

Aging had a negative effect on the structure and reactivity of Febased nanoparticles toward target contaminants. ${ }^{1}$ The reducibility of the S-nZVI synthesized in this study was found to decrease obviously, as indicated by the low NB removal and AN formation efficiency after aging for 12 weeks (Fig. 6a). Similar phenomenon regarding the obvious loss of reactivity have been reported in previous studies. For example, Su et al. ${ }^{8}$ found that S-nZVI retained $60.7 \%$ of its original cadmium removal capacity after exposure for 14 weeks. Li et al. ${ }^{1}$ observed that eleven weeks old S-nZVI could maintain approximately $55 \%$ of the original capacity for tetrabromobisphenol degradation. In contrast, SnZVI@BC prepared in this study retained high NB reduction reactivity after aging for 12 weeks, as indicated by NB removal efficiency as high as $72.2 \pm 1.8 \%$ and AN formation efficiency as high as $31.7 \pm 2.1 \%$ after aging. Thus, it could be inferred that the antioxidation ability of S-nZVI@BC was superior in comparison to bare S-nZVI probably due to the fact that the nanoscale S-nZVI particles were partially loaded in the mesoporous BC channels (Fig. 1c). Due to the protection of BC channels, the contact of oxygen could be effectively reduced and thus the longevity of S-nZVI@BC could be extended.

Undoubtedly, reusability was one of the most significant issues that determined the practical applicability of nZVI-based nanoparticles for the removal of target contaminants. As shown in Fig. 6b, NB removal and AN formation efficiency of S-nZVI reduced significantly from $70.0 \pm 4.2 \%$ and $49.4 \pm 1.4 \%$ at the first cycle to $21.8 \pm 3.4 \%$ and $0 \%$ at the fourth cycle. However, the NB removal and AN formation efficiency of SnZVI@BC decreased slowly from $100 \%$ and $84.1 \pm 2.1 \%$ at the first cycle to $72.2 \pm 2.3 \%$ and $25.0 \pm 1.2 \%$ at the fourth cycle, indicating rather high reusability of S-nZVI@BC towards NB transformation. Similar findings have also been reported for other nZVI-supported materials used for various pollutants removal. For example, Li $e t a l .^{34}$ reported that NB removal efficiency of clay supported nZVI was $42.7 \pm 2.8 \%$ in the fifth cycle, whereas as low as almost $10 \%$ for bare nZVI. They pointed that the immobilized nZVI particles prevented their agglomeration and improved the reusability of the clay supported nZVI. In this study, the decrease in NB removal efficiency of S-nZVI@BC after reuse could be attributed to consumption of S-nZVI, barrier effect of the corrosion products and adsorption saturation of NB on the surface of S-nZVI@BC. (a)

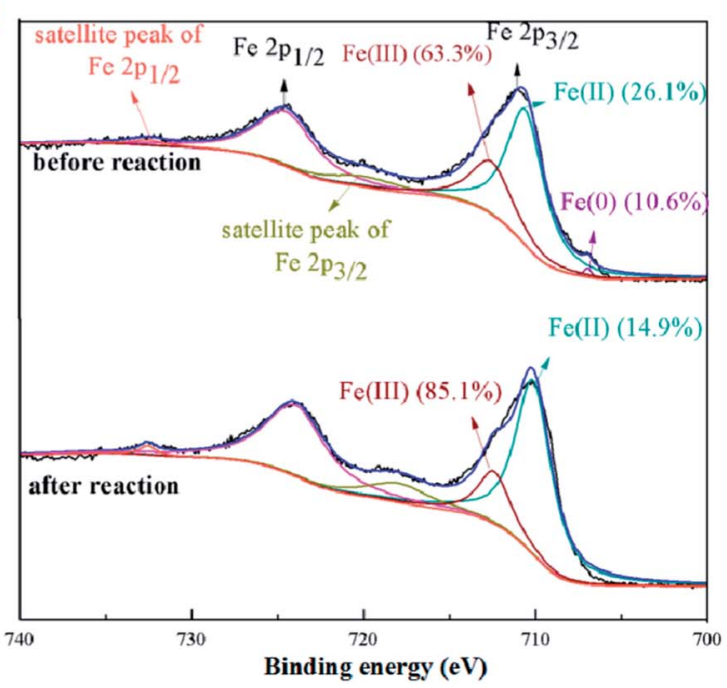

(b)

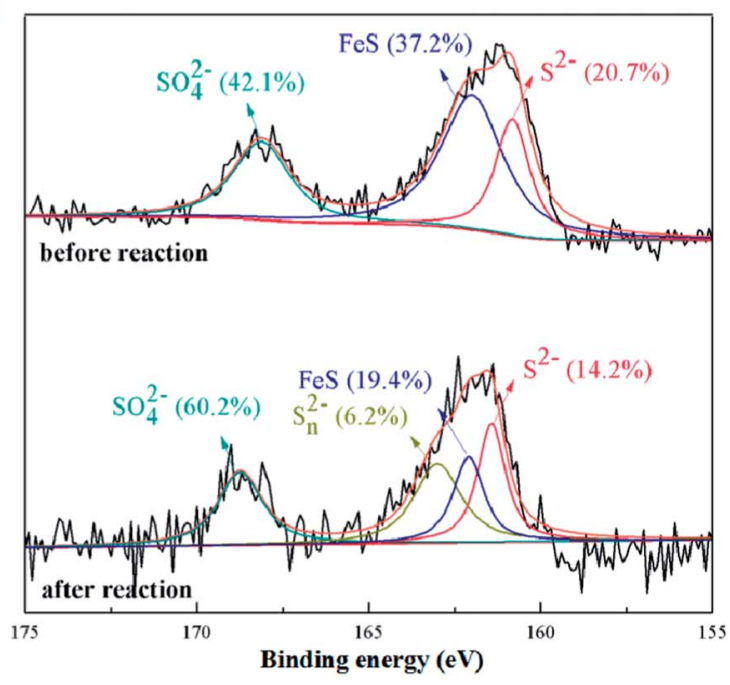

Fig. 5 XPS spectra of Fe $2 p$ region (a) and S $2 p$ region (b) of S-nZVIaBC before and after reaction with NB. 
(a)
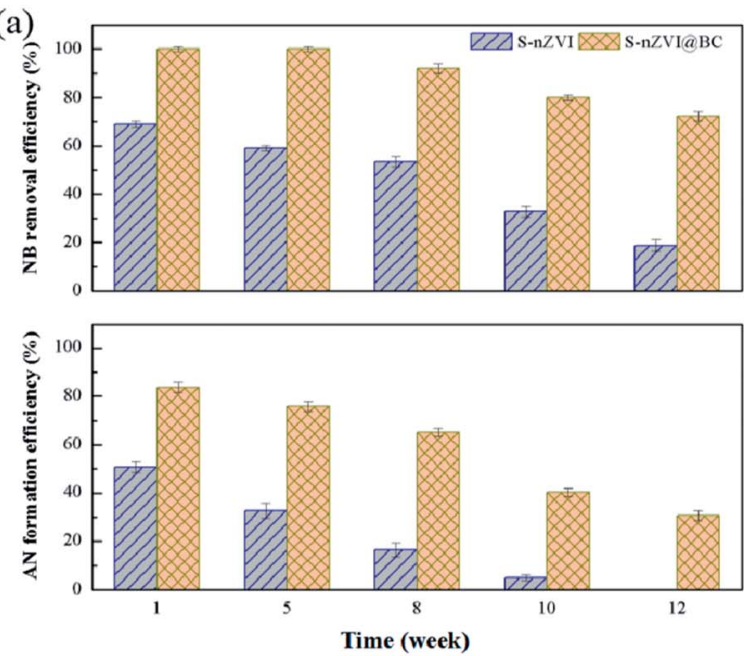

(b)
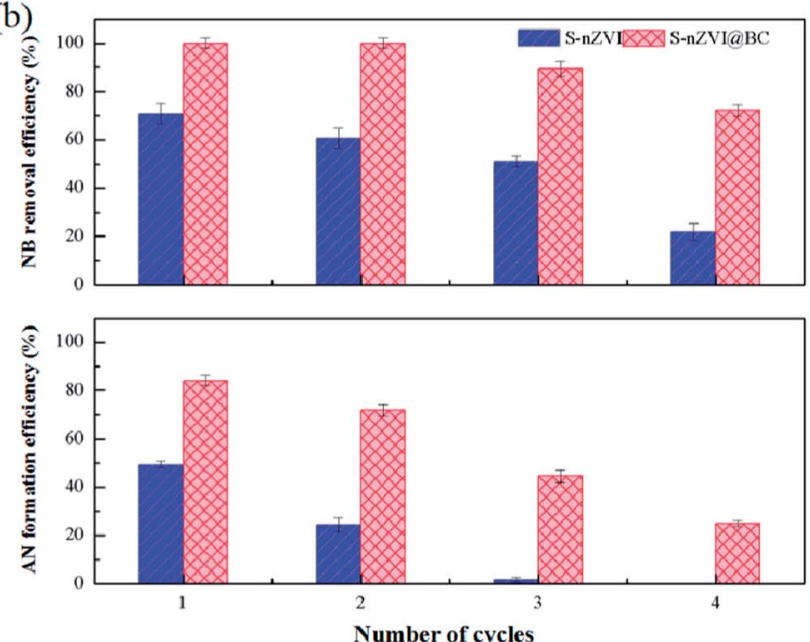

Fig. 6 The effects of aging (a) and reusability (b) on NB removal and AN formation by S-nZVI and S-nZVI@BC.

\subsection{Synergistic mechanism for NB removal by S-nZVI@BC}

In order to figure out the synergistic mechanism for NB removal by S-nZVI@BC, the performance of S-nZVI@BC, S-nZVI and BC was compared. Since the mass ratio of S-nZVI and BC in SnZVI@BC was approximately $3: 1$, dosages of S-nZVI@BC, SnZVI and BC were controlled at $1.00 \mathrm{~g} \mathrm{~L}^{-1}, 0.75 \mathrm{~g} \mathrm{~L}^{-1}$ and $0.25 \mathrm{~g} \mathrm{~L}^{-1}$, respectively. As shown in Fig. S5, $\uparrow 200 \mathrm{mg} \mathrm{L}^{-1} \mathrm{NB}$ could be efficiently removed by the dosage of $1.00 \mathrm{~g} \mathrm{~L}^{-1} \mathrm{~S}$ nZVI@BC, with NB removal and AN formation efficiency as high as $88.7 \pm 3.0 \%$ and $53.2 \pm 3.3 \%$ after $90 \mathrm{~min}$. However, with the dosage of $0.25 \mathrm{~g} \mathrm{~L}^{-1} \mathrm{BC}$ and $0.75 \mathrm{~g} \mathrm{~L}^{-1} \mathrm{~S}-\mathrm{nZVI}, \mathrm{NB}$ removal and AN formation efficiency was as low as $4.9 \pm 0.8 \%$ and $0 \%, 32.7 \pm 3.2 \%$ and $13.2 \pm 1.2 \%$, respectively. With the simultaneous addition of $0.75 \mathrm{~g} \mathrm{~L}^{-1} \mathrm{~S}-\mathrm{nZVI}$ and $0.25 \mathrm{~g} \mathrm{~L}^{-1} \mathrm{BC}$, NB removal and AN formation efficiency were $42.7 \pm 2.4 \%$ and $22.4 \pm 1.7 \%$, which was significantly lower than those in the degradation system by S-nZVI@BC. These results illustrated the synergistic effect between S-nZVI and BC for NB removal and AN formation. Similar phenomena have been reported in previous studies, where BC supported nanoscale iron sulfide composite showed significantly higher $\mathrm{Cr}(\mathrm{vI})$ removal efficiency than the sum of blank BC, FeS and carboxymethyl cellulose. ${ }^{19}$

Based on the above analysis, it could be inferred that mechanism such as the chemical adsorption, redox reactions and enhanced electron transfer could be involved in the NB removal by S-nZVI@BC, as indicated in Fig. 7. The BC support material not only prevented the agglomeration of S-nZVI particles, but also promoted NB adsorption due to the large specific surface area and rich functional groups. According to previous studies, NB could be easily reduced by direct electron donation of nZVI, ferrous irons and sulfide. ${ }^{35-37}$ Moreover, the enhanced electron transfer between S-nZVI@BC and the adsorbed NB facilitated NB transformation, considering the excellent electrical conductivity of FeS on nZVI surface. ${ }^{1}$ In addition, Oh et $a .^{38}$ found that the BC addition strikingly accelerated the transformation of redox-sensitive nitro compounds, which could be attributed to the enhanced electron transfer by BC. As shown in Fig. S6a, $\uparrow$ the redox peaks of glassy carbon electrode

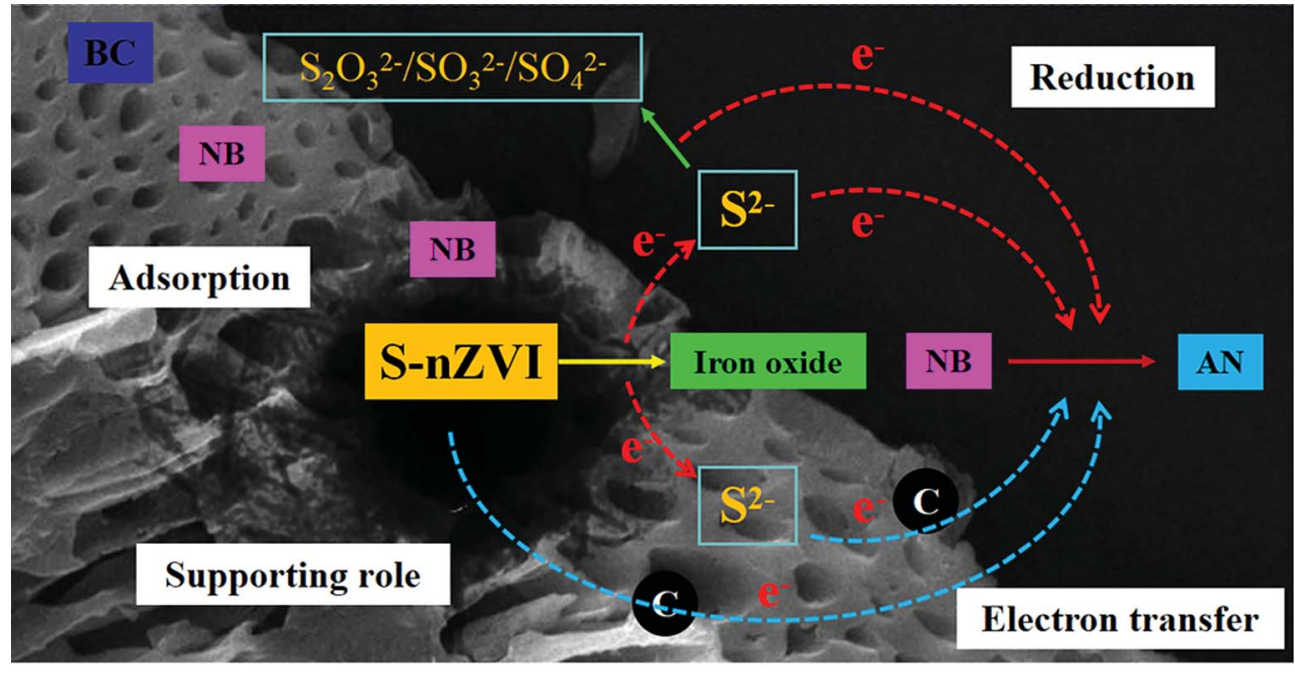

Fig. 7 Proposed mechanism for enhanced NB removal by S-nZVIaBC. 
(GCE) modified by S-nZVI@BC had a higher current density than GCE modified by S-nZVI. Furthermore, the typical Nyquist plots showed that S-nZVI@BC-modified GCE had a smaller semicircle diameter but a lower $Z^{\prime \prime}$ value at a high frequency compared to that of S-nZVI-modified GCE (Fig. S6b $\dagger$ ), confirming the key role of $\mathrm{BC}$ in electron transfer between NB and SnZVI. ${ }^{39}$ Therefore, the synergistic mechanism involved in NB removal by S-nZVI@BC mainly included the following aspects: (a) chemical adsorption of NB onto S-nZVI@BC via functional groups and surface pores, (b) reduction of NB by S-nZVI and (c) electron transfer by S-nZVI@BC.

\section{Conclusions}

In the present study, a highly efficient reductant was successfully synthesized through loading nanoscale S-nZVI composite onto BC. NB removal and AN formation of S-nZVI@BC could be significantly enhanced, as compared to S-nZVI and blank BC. NB removal by S-nZVI@BC followed second-order kinetics model and Langmuir isotherm model, indicating the involvement of hybrid chemical reaction-adsorption process. Synergistic mechanism, including chemical adsorption of NB onto SnZVI@BC, direct reduction by S-nZVI and enhanced electron transfer, was responsible for the enhanced NB removal by SnZVI@BC. The excellent reducibility of S-nZVI@BC makes it to be a promising alternative for the remediation of sites contaminated by NB.

\section{Conflicts of interest}

There are no conflicts to declare.

\section{Acknowledgements}

This work was supported by the Natural Science Foundation of Jiangsu Province for Distinguished Young Scholars (BK20170038), the National Natural Science Foundation of China (51778294 and 51708293) and the Natural Science Foundation of Jiangsu Province (BK20170842).

\section{References}

1 D. Li, Z. Mao, Y. Zhong, W. L. Huang, Y. D. Wu and P. A. Peng, Water Res., 2016, 103, 1-9.

2 Y. Xu and M. A. A. Schoonen, Am. Mineral., 2000, 85, 543-556. 3 R. G. Pearson, Inorg. Chem., 1988, 27, 734-740.

4 S. R. C. Rajajayavel and S. Ghoshal, Water Res., 2015, 78, 144153.

5 E. J. Kim, K. Murugesan, J. H. Kim, P. G. Tratnyek and Y. S. Chang, Ind. Eng. Chem. Res., 2013, 52, 9343-9350.

6 C. Levard, E. M. Hotze, B. P. Colman, A. L. Dale, L. Truong, X. Y. Yang, A. J. Bone, G. E. Brown, R. L. Tanguay, R. T. Di Giulio, E. S. Bernhardt, J. N. Meyer, M. R. Wiesner and G. V. Lowry, Environ. Sci. Technol., 2013, 47, 13440-13448.

7 E. J. Kim, J. H. Kim, A. M. Azad and Y. S. Chang, ACS Appl. Mater. Interfaces, 2011, 3, 1457-1462.
8 Y. M. Su, A. S. Adeleye, A. A. Keller, Y. X. Huang, C. M. Dai, X. F. Zhou and Y. L. Zhang, Water Res., 2015, 74, 47-57.

9 C. J. Ou, J. Y. Shen, S. Zhang, Y. Mu, W. Q. Han, X. Y. Sun, J. S. Li and L. J. Wang, Water Res., 2016, 101, 457-466.

10 S. A. Messele, O. S. G. P. Soares, J. J. M. Orfao, C. Bengoa, F. Stuber, A. Fortuny, A. Fabregat and J. Font, Catal. Today, 2015, 240, 73-79.

11 X. G. Li, Y. Zhao, B. D. Xi, X. G. Meng, B. Gong, R. Li, X. Peng and H. L. Liu, J. Environ. Sci., 2017, 52, 8-17.

12 H. Y. Shu, M. C. Chang, C. C. Chen and P. E. Chen, J. Hazard. Mater., 2010, 184, 499-505.

13 Faheem, H. X. Yu, J. Liu, J. Y. Shen, X. Y. Sun, J. S. Li and L. J. Wang, J. Taiwan Inst. Chem. Eng., 2016, 66, 313-320.

14 J. M. Dias, M. C. M. Alvim-Ferraz, M. F. Almeida, J. RiveraUtrilla and M. Sanchez-Polo, J. Environ. Manage., 2007, 85, 833-846.

15 Q. Y. Hu, Y. L. Zhu, B. W. Hu, S. H. Lu and G. D. Sheng, J. Environ. Sci., 2018, DOI: 10.1016/j.jes.2018.01.013.

16 M. B. Ahmed, J. L. Zhou, H. H. Ngo, W. S. Guo, M. A. H. Johir, K. Sornalingam, D. Belhaj and M. Kallel, Chem. Eng. J., 2017, 322, 571-581.

17 D. J. Zhang, W. Li, C. Hou, J. Y. Shen, X. B. Jiang, X. Y. Sun, J. S. Li, W. Q. Han, L. J. Wang and X. D. Liu, Chem. Eng. J., 2017, 314, 88-97.

18 M. Y. Yen, C. C. Teng, M. C. Hsiao, P. I. Liu, W. P. Chuang, C. C. M. Ma, C. K. Hsieh, M. C. Tsai and C. H. Tsai, J. Mater. Chem., 2011, 21, 12880-12888.

19 H. H. Lyu, J. C. Tang, Y. Huang, L. S. Gai, E. Y. Zeng, K. Liber and Y. Y. Gong, Chem. Eng. J., 2017, 322, 516-524.

20 M. B. Ahmed, J. L. Zhou, H. H. Ngo, W. S. Guo, M. A. Johir and K. Sornalingam, Chem. Eng. J., 2017, 311, 348-358.

21 J. K. Du, J. G. Bao, C. H. Lu and D. Werner, Water Res., 2016, 102, 73-81.

22 R. Yang, Y. Wang, M. Li and Y. J. Hong, ACS Sustainable Chem. Eng., 2014, 2, 1270-1279.

23 Y. Mu, R. A. Rozendal, K. Rabaey and J. Keller, Environ. Sci. Technol., 2009, 43, 8690-8695.

24 W. L. Sun, W. Y. Pan, F. Wang and N. Xu, Chem. Eng. J., 2015, 273, 353-362.

25 S. Gupta and B. V. Babu, Chem. Eng. J., 2009, 150, 352-365.

26 R. Mantha, K. E. Taylor, N. Biswas and J. K. Bewtra, Environ. Sci. Technol., 2001, 35, 3231-3236.

27 Y. Sun, Z. M. Lou, J. B. Yu, X. X. Zhou, D. Lv, J. S. Zhou, S. A. Baig and X. H. Xu, Chem. Eng. J., 2017, 323, 483-491.

28 Y. L. Zhu, C. Zheng, S. Y. Wu, Y. Z. Song and B. W. Hu, J. Radioanal. Nucl. Chem., 2018, 316, 1337-1346.

29 X. Peng, B. D. Xi, Y. Zhao, Q. T. Shi, X. G. Meng, X. H. Mao, Y. H. Jiang, Z. F. Ma, W. B. Tan, H. L. Liu and B. Gong, Environ. Sci. Technol., 2017, 51, 10100-10108.

30 M. Q. Qiu, M. Wang, Q. Z. Zhao, B. W. Hu and Y. L. Zhu, Chemosphere, 2018, 201, 764-771.

31 C. F. Wang, D. Yao, Y. Liu, Y. F. Wu and J. Y. Shen, Waste Manage., 2018, 76, 679-686.

32 T. Yamashita and P. Hayes, Appl. Surf. Sci., 2008, 254, 24412449.

33 Q. A. Tian, Q. A. Wang, Q. S. Xie and J. G. Li, Nanoscale Res. Lett., 2010, 5, 1518-1523. 
34 X. G. Li, Y. Zhao, B. D. Xi, X. H. Mao, B. Gong, R. Li, X. Ping and H. L. Liu, Appl. Surf. Sci., 2016, 370, 260-269.

35 B. Liang, H. Y. Cheng, D. Y. Kong, S. H. Gao, F. Sun, D. Cui, F. Y. Kong, A. J. Zhou, W. Z. Liu, N. Q. Ren, W. M. Wu, A. J. Wang and D. J. Lee, Environ. Sci. Technol., 2013, 47, 5353-5361.

36 X. D. Yu, W. W. Gong, X. H. Liu, L. Shi, X. Han and H. Y. Bao, J. Hazard. Mater., 2011, 198, 340-346.
37 J. Dong, L. J. Ding, Z. F. Chi, J. S. Lei and Y. Su, J. Environ. Sci., 2017, 54, 206-216.

38 S. Y. Oh, J. G. Son and P. C. Chiu, Environ. Earth Sci., 2015, 73, 1813-1822.

39 D. Zheng, J. S. Ye, L. Zhou, Y. Zhang and C. Z. Yu, Electroanalysis, 2009, 21, 184-189. 